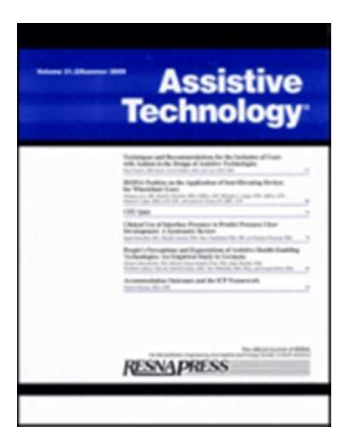

\title{
Engaging Older Adults with Dementia in Creative Occupations Using Artificially Intelligent Assistive Technology
}

\begin{tabular}{|r|l|}
\hline Journal: & Assistive Technology \\
\hline Manuscript ID: & UATY-2012-0409.R1 \\
\hline Manuscript Type: & Original Scientific Article \\
\hline Keywords: & $\begin{array}{l}\text { Assistive technology, Human-computer interface, Intelligent systems, } \\
\text { Memory impairment, Quality of life, Older adults }\end{array}$ \\
\hline \multicolumn{2}{|l}{} \\
\hline
\end{tabular}

SCHOLARONE ${ }^{\text {m }}$

Manuscripts 
Running Head: ENGAGEMENT IN CREATIVE OCCUPATIONS USING ARTIFICIALLY INTELLIGENT TECHNOLOGY

\section{Abstract}

2 Engagement in creative occupations has been shown to promote well-being for older adults with

3 dementia. Providing access to such occupations is often difficult as successful participation

4 requires face-time with a person who is knowledgeable in facilitating engagement as well as

5 access to any required resources, such as an arts studio. In response, a computer-based device,

6 the Engaging Platform for Art Development (ePAD), was created to with the aim of enabling

7 more independent access to art creation. ePAD is a an artificially intelligent touch-screen device

8 that estimates a client's level of engagement and provides prompts to encourage engagement if

9 the client becomes disengaged. ePAD is customizable such that an art therapist can choose

10 themes and tools that they feel reflect their client's needs and preferences. This paper presents a

11 mixed-methods study that evaluated ePAD's usability by six older adult (with mild-to-moderate

12 dementia) and art therapist dyads. Usability measures suggest that all participants found ePAD

13 engaging but did not find prompts effective. Future development of ePAD includes improving

14 the prompts, implementing the recommendations made by participants in this research, and long-

15 term testing in more naturalistic art therapy contexts.

16

17 Key words: Assistive technology, Human-computer interface, Intelligent systems, Memory

18 impairment, Quality of life, Older adults. 
ENGAGEMENT IN CREATIVE OCCUPATIONS USING ARTIFICIALLY INTELLIGENT TECHNOLOGY

\section{Introduction}

For people with dementia, participation in a meaningful occupation can promote quality

3 of life (QoL), facilitate communication and emotional expression, and enhance personal

4 relationships (Rusted, Sheppard, \& Waller, 2006; Stoukides, 2008). This perspective

5 complements that of the American Art Therapy Association (2011), which defines art therapy as

6 "a mental health profession that uses the creative process of art making to improve and enhance

7 the physical, mental and emotional well-being of individuals of all ages".

8 The literature indicates that individuals with dementia may benefit from participation in

9 creative processes (Karp, et al., 2006; Waller, 2002). However, these individuals may have

10 difficulty engaging in various occupations for a number of different reasons, including

11 diminished desire to participate in an activity and a reduced ability to self-engage in a task

12 (Lubinski, 1991). Computer-based technology has the potential to help overcome many of these

13 limitations by providing an interactive structure that could foster participation in art activities, as

14 well as technique for monitoring participants' levels of engagement (Carrillo, Dishman, \&

15 Plowman, 2009). In a treatment setting, this approach could be used for therapeutic and

16 diagnostic purposes.

17 Advances in technology have expanded the media available to art therapists (hereafter

18 referred to as therapists). Computer-based art programs allow therapists to manage many aspects

19 of the artwork and art-creating process (Mattson, 2010), allow for multiple reproductions of an

20 image for manipulation or re-creation (Orr, 2005), and can be adapted to compensate for a lack

21 of cognitive or physical skills necessary for more traditional art mediums (Kuleba, 2008). For

22 example, in a case study by Cummings, Miller, Christensen, and Cherry (2008), a client with a 
ENGAGEMENT IN CREATIVE OCCUPATIONS USING ARTIFICIALLY INTELLIGENT TECHNOLOGY

1 degenerative neurological disease was able to communicate and continue her previous

2 occupation of painting using a computer system that tracked eye movements.

3 Despite the benefits of integrating computer technology into art therapy, therapists have

4 noted several limitations, including a lack of training and experience with computer hardware

5 and software (Kuleba, 2008). Moreover, therapists have expressed concern surrounding the lack

6 of sensory qualities of using a computer to create artwork and the cost of integrating these

7 technologies into practice (Kuleba, 2008; Orr, 2005). Additionally, therapists have described

8 emotional factors, such as their own technology-related anxiety, as a barrier for integrating

9 computers in practice (Peterson, 2010). While a large proportion of therapists report use of some

10 form of technology to support their profession, very few report using technology as a therapeutic

11 medium (Orr, 2005). A properly designed computer-based device could allow a therapist to

12 capitalize on the time spent with each client with dementia during a session while promoting

13 autonomy in the creation of art (Blunsden, et al., 2009; Mihailidis, et al., 2010). There is

14 potential for such a device to support group art therapy sessions by providing some measure of

15 semi-autonomous support to each client, thus lessening individual clients' demands for artistic

16 assistance from the therapist.

\section{Overview of the Engaging Platform for Art Development (ePAD)}

18 The authors developed a computer-based art device: the Engaging Platform for Art

19 Development (ePAD; Figure 1) with the goals of engaging persons with dementia in creative art

20 occupations, providing appropriate structure and cues to engage these persons, and to mitigate

21 barriers to using technology in art therapy contexts. ePAD uses artificial intelligence (i.e.,

22 software that allows a computer to make human-like decisions) to monitor a client's (i.e., an

23 older adult with dementia) level of engagement in order that ePAD can actively engage the client 
1 in an art activity, using prompts should the client become disengaged (Blunsden, et al., 2009;

2 Mihailidis, et al., 2010). This is accomplished by tracking how much the client is touching the

3 screen and by tracking whether or not the client is looking at the screen, as described in Blunsden

4 et al. (2009). The first ePAD prototype was developed from information gathered by an

5 international online survey and subsequent focus group with therapists who were working with

6 older adults with dementia (Blunsden, et al., 2009; Mihailidis, et al., 2010). Seven therapists

7 evaluated the prototype and offered suggestions for improvement (Hoey, Zutis, Leuty, \&

8 Mihailidis, 2010), which were incorporated into the version of ePAD used in the research

9 presented in this paper.

10 The therapist uses ePAD's touch screen (top of Figure 1b) to design the layout of the

11 screen that will be used by their client (bottom of Figure 1b). This includes selecting a

12 background for the canvas area (which could be a solid colour or an image for the client to use as

13 a starting point), selecting the tools that will be used by the client, configuring the features and

14 functions of these tools, and selecting the prompts ePAD will use to engage the client. Tools can

15 are represented by an image that depicts what the tool does. For example, there is a tool that

16 looks like a paintbrush that the client can use to "paint" with and there are tools that look like

17 shapes that the client can touch and then "stamp" onto the canvas area. Prior to a session, the

18 therapist sets up a client's toolbox by selecting the desired tools from the available tools on the

19 left side of the screen and dragging them onto the toolbox area (top Figure 1b). The therapist can

20 then configure the tool through simple commands using a mouse (e.g. resize the tool by clicking-

21 and-dragging). Additionally, there is a properties menu that allows the therapist to configure

22 options specifically related to each tool, such as background colour, changing the tool's icon, a

23 label for the tool, and other functionality options. The therapist can therefore make the 
ENGAGEMENT IN CREATIVE OCCUPATIONS USING ARTIFICIALLY INTELLIGENT TECHNOLOGY

1 application as simple or as complex as he/she wishes for a particular client. When a client is

2 using ePAD, only the canvas and toolbox are visible (bottom of Figure $1 \mathrm{~b}$ ) and tools' properties

3 cannot be changed. The client interacts with ePAD by selecting (or having the therapist help

4 them select) tools and then touching the canvas area on ePAD's screen. The client can use their

5 fingers or an object, such as a paintbrush, to interact with ePAD.

6 When a client is working with ePAD, ePAD autonomously estimates a client's level of

7 engagement based on the client's interactions with the device and whether or not he/she is

8 looking at the screen. This estimate of engagement allows ePAD to provide the client with

9 appropriate prompts. For example, if ePAD senses that the client's engagement level is dropping,

10 it can issue a prompt to try to re-engage the client. ePAD's prompts include adding shapes or

11 images to the canvas, and animating tools to attract the client's attention. The images and audio

12 used by the prompts are fully customizable by the therapist. The therapist can also set the

13 threshold engagement level; when ePAD estimates the user's engagement is below this

14 threshold, ePAD will begin prompting the client using the prompts that were specified by the 15 therapist.

\section{Purpose}

17 The purpose of the research was to test ePAD with dyads of art therapists (therapists) and 18 older adult (OA) clients with mild-to-moderate dementia. This research investigated the

19 following research questions:

20 1. What is the usability of ePAD for therapists who work with OAs diagnosed with $21 \quad$ mild-to-moderate dementia?

22 2. What is the usability of ePAD for OAs diagnosed with mild-to-moderate dementia in $23 \quad$ an individual art therapy context? 
ENGAGEMENT IN CREATIVE OCCUPATIONS USING ARTIFICIALLY INTELLIGENT TECHNOLOGY

1

2 Method

3 Research Design

4

5

6

7

8

10

11

12 clients with dementia thought about $\mathrm{PAD}$ and to identify areas that could use improvement. and

13 used a pragmatic mixed methods, concurrent nested design, where quantitative and qualitative

14 data were collected and analyzed concurrently but with unequal priority; qualitative data (written

15 comments) were only used to clarify quantitative results (Hanson, Creswell, Clark, Petska, \&

16 Creswell, 2005). Quantitative data was obtained through questionnaires that used five-point

17 Likert rating scales which measured ePAD's usability from the perspectives of therapists and

18 OAs .

\section{Participants}

20 Art therapist participants had to have at least two years of experience working with OAs

21 with dementia in art therapy. OA participants had to be over 65 years in age, have mild-to-

22 moderate dementia (represented by a Mini-Mental State Examination (MMSE) score of 10 to 24

URL: http://mc.manuscriptcentral.com/uaty Email: journal@resna.org 
ENGAGEMENT IN CREATIVE OCCUPATIONS USING ARTIFICIALLY INTELLIGENT TECHNOLOGY

1 out of 30 (Folstein, Folstein, \& McHugh, 1975)), be physically able to participate, and be

2 currently participating in an art therapy program at [FACILITY MASKED]. Study approval was

3 obtained from the Research Ethics Boards of [FACILITY NAME MASKED], [INSTITUTION

4 MASKED], and [INSTITUTION MASKED]. Consent was obtained from all therapist

5 participants and OAs' surrogate decision makers prior to starting the trials.

\section{$6 \quad$ Study Procedures}

7 Each therapist was introduced to ePAD through a one hour, one-on-one training session

8 with a researcher. Therapists were encouraged to explore ePAD's features and then to set up

9 ePAD for the specific client they would be working with. This included setting up their client's

10 canvas and toolbox as well as selecting and customizing the prompts ePAD would use to try and

11 engage the client if he or she became disengaged. For the trials described in this work, there were

12 approximately 80 tools available, which included a paint brush, different shapes or "stamps", and

13 images that could be placed and resized. Therapists were encouraged to make any changes they

14 wished to ePAD's setup between sessions.

15 Up to five, one-hour trials were held with each therapist-OA dyad in the Creative Arts

16 Therapies studio at [FACILITY MASKED]. Each trial began with the therapist explaining the

17 goal of the session to their client. The OA was then encouraged to interact with ePAD as

18 independently as possible. Throughout each trial, ePAD automatically determined the OA's

19 engagement every ten seconds and the research assistant made observational notes. At the end of

20 each trial, therapists and OAs completed a questionnaire regarding their perceptions of ePAD.

21 All questionnaires were administered verbally to OAs and in writing for therapists.

22 Questionnaires can be viewed at:

23 http://www.ot.utoronto.ca/iatsl/documents/epad_questionnaires.pdf. After each trial, therapists 
ENGAGEMENT IN CREATIVE OCCUPATIONS USING ARTIFICIALLY INTELLIGENT TECHNOLOGY

1 were encouraged to make any changes to ePAD's setup that they thought would be appropriate

2 for the next session. All sessions were recorded by ePAD, enabling the therapist to consult logs

3 of previous sessions. Each log contained information collected every 30 seconds throughout the

4 related session, including ePAD's estimates of engagement levels, screenshots of the client's

5 artwork, and ePAD's actions. By reviewing a log, the therapist could review what happened

6 during the session and print off any information or pictures that were of interest.

\section{Data Analysis}

8 Median responses from Likert questions were calculated from questionnaire responses.

9 A directed content analysis was conducted on the qualitative data gathered from participants'

10 comments (both therapists and OAs) using coding categories and subcategories that were

11 developed by the research team from the quantitative data. The size of the data samples allowed

12 for a descriptive statistical analysis.

\section{Results}

\section{Participants}

15 Art therapist participants $(n=6)$ had at least two years of clinical experience working with 16 OAs with dementia. OA participants $(n=6$, mean age $=89.2$ years $)$ had a mean MMSE score of

$1716.5(\min =15, \max =25)$. Of the six therapist-OA dyads, only three completed all five trials; one

18 OA passed away after the second trial, one became ill after completing three trials, and one

19 found the experience overwhelming and chose to withdraw after the first trial. 
ENGAGEMENT IN CREATIVE OCCUPATIONS USING ARTIFICIALLY INTELLIGENT TECHNOLOGY

\section{Usability}

2 Figures 2 to 4 present the post-session questionnaire Likert data for therapist and OA

3 participants. The subsequent sub-sections present summaries of the coded analysis of the

4 qualitative data used to explore different aspects of ePAD's usability.

\section{Efficiency}

6 Ease of use according to therapists. Overall, therapists reported configuring the system

7 to accommodate the needs of a specific client as being fairly intuitive, however, half of the

8 therapists expressed some discomfort in navigating the functions and/or fear of potential

9 problems with the system. For example, one therapist stated, "The system was relatively easy to

10 set up; if there was a problem though, I'd be lost as to how to fix it!” [AT6].

11 Therapists felt selecting and configuring system actions and additional settings was fairly

12 straightforward. The exception was setting up the level of prompting, which was described as

13 being confusing by two of the therapists, who felt that the wording describing the prompting

14 levels were unclear and the scales used to determine when ePAD would prompt the client were

15 counter-intuitive. Two therapists felt that larger text and/or more visually appealing tools would

16 have aided in finding the menus for system actions and settings tabs. When choosing how each

17 tool would look, two therapists commented that better graphics would likely make this system

18 more intuitive for clients, for example, one therapist commented "There was an icon for the

19 paintbrush, but it would have been nice to have additional icons for the pen, paint, etc. which

20 looked like a pen, etc [AT5]."

21 A "Setup Wizard" was available to help therapists learn about the device and assist with the 22 set up process, however, during four of the training sessions this tool did not work properly. 
ENGAGEMENT IN CREATIVE OCCUPATIONS USING ARTIFICIALLY INTELLIGENT TECHNOLOGY

1 Therapists seemed interested in the logs captured by ePAD, however, no therapist asked to see

2 their client's logs; they only looked at them when prompted to do so by the researcher.

3 Ease of use according to OAs. Four of the OAs expressed excitement about the novelty of

4 the device and the ease of using specific features, such as, "It was hard at first but then got easy

5 with practice. Creating the art was good. At least you are doing something with your hands and

6 your mind [OA2]." OAs had suggestions for increasing ePAD's efficiency, such as, "If I wanted

7 [a specific] effect, it was hard. I would have needed different types and widths of brushes...it

8 made marks I didn't like because I didn't have that feeling of a real brush. It was a puzzlement to

9 me; I felt at a complete loss as to what effect I would get from the brush [OA3]."

10 Assistance needed from a therapist. While ePAD was intended to support art therapy in a

11 group setting, four therapists commented that they did not feel that using ePAD would allow

12 them to spend more time with other clients if it were used in a group setting. This was reflected

13 in the average "disagree" responses to questions regarding use in a group (Figure 3) and

14 comments such as, "There were times when the device was quite efficient; [my] client was able

15 to use it without prompting. At other times, though, the buttons and functions required quite a bit

16 of explanation, which required some time and repetition [AT6]." Overall, therapists felt ePAD

17 might distract their other clients in a group setting and felt they needed to provide a fair amount

18 of guidance to their client when using ePAD in the trials. Furthermore, therapists emphasised

19 that the relationship OAs develop through interactions with a therapist is an integral part of the

20 art therapy process.

\section{Effectiveness}

22 Ability to Engage. Five therapists felt ePAD was a useful tool for engaging clients in a

23 creative process. All of the therapists felt there were sufficient system actions and that the 
ENGAGEMENT IN CREATIVE OCCUPATIONS USING ARTIFICIALLY INTELLIGENT TECHNOLOGY

1 available settings were helpful in configuring the device for specific clients. Three therapists had

2 suggestions for improving device settings, including "When setting background pictures, the

3 sizing needs to be adjustable [AT2]," and, "Textured backgrounds would be an asset (i.e.,

4 canvas, paper, wood grain, etc.) [AT4]."

5 Prompting. Very few prompts were given by ePAD and all therapists felt the device was

6 ineffective in prompting clients. OAs indicated that prompts were not attention grabbing. On the

7 occasions an OA acknowledged a prompt, they generally did not react in the intended fashion.

8 Satisfaction

9 ePAD's design. Overall, all therapists were satisfied with ePAD's design, including the

10 form-factor of an easel, representation of paint colours, and selection of tools. Four therapists felt

11 that the screen needed to be altered, such as, "The vision of my client is already compromised, so

12 crisper lines [on the screen] would be better [AT5]," and, "The easel seemed very big/bulky. I

13 would like it to tilt with ease if possible. The screen sensitivity was fantastic! [AT3]."

14 Therapists were generally satisfied with how ePAD operated and could envision using the

15 device with clients. Comments included, "[My client] was interested, curious, and had many

16 questions/ comments. The device is quiet, clean, and easy to use. Curiosity was sparked and he

17 arrived at a project idea easily [AT3]," and, "I was surprised at how long the client remained

18 engaged. It was a positive experience for him [AT5].” Therapists also offered suggestions for

19 improvement of ePAD's functionality, including, "Multiples of an image appearing when the

20 client accidentally touches the screen two or three times is distracting [to] have to undo. It would

21 be better if the client had to touch an image again in order for it to appear again [AT2]."

22 Ability to achieve engagement in art. Therapists seemed uncertain as to whether they or

23 their clients were satisfied in using the device to create art. OA participants reported high 
1 satisfaction with ePAD and its novelty. They stated that they enjoyed working with the device

2 and were pleased with the art they were able to create, expressed by comments such as, "It's

3 fantastic. It's miles ahead of anything I've ever seen to give you an ability to do something

4 [OA3]." OAs were not able to report on satisfaction with prompts because they did not recall that

5 the device had prompted them or did not notice the prompts when they played.

6 Discussion

$7 \quad$ During the training session, therapists expressed minimal or no anxiety about using

8 ePAD, seemed to be able to set up and configure the system easily, and reported that customizing

9 ePAD was relatively intuitive. However, during the subsequent trials, therapists requested a

10 significant amount of support from the researcher and expressed that navigating setup and post-

11 trial configuration modifications caused some discomfort. This perception did not appear to

12 change over the course of the study, however, as there were only five trials (which were usually

13 held once per week) this perception might diminish with greater exposure.

14 Alterations to the setup and configuration process would likely improve ePAD's

15 usability. Therapists gave valuable suggestions, such as including visuals in the set up process

16 and more intuitive wording of features' descriptions. Improvements to the Setup Wizard, such as

17 ensuring it works robustly, increasing visuals and verbal prompts, and rewording menu items and

18 function descriptions would likely improve usability and feelings of mastery. With regard to the

19 physical design of ePAD, the fixed angle of the screen was problematic for some clients; those

20 who had a limited range of motion had trouble reaching the canvas. Originally the screen's

21 height and angle could be adjusted with little effort, however, to ensure stability, the structure

22 was reinforced in such a way that the height and angle became fixed; this needs to be remedied

23 in future versions. 
ENGAGEMENT IN CREATIVE OCCUPATIONS USING ARTIFICIALLY INTELLIGENT TECHNOLOGY

1

2

3

4 as intended, it is difficult to truly gauge this aspect. Prompting improvements include more

5 detailed verbal prompts, such as addressing the client by name, and more distinctive, eye-

6 catching visual prompts to get the client's attention. Research similar to Wilson et al. (Wilson,

7 Rochon, Mihailidis, \& Leonard, 2012) would be required to develop effective and appropriate

8 prompting strategies. Moreover, careful consideration is needed regarding how, when, and what

9 prompts should be employed if ePAD is to be used in a group therapy setting.

While the logs of client's interactions with ePAD were interesting to the therapists, it seems more work needs to be done to identify potential uses of this functionality as well as to ensure data is presented in an accessible and useful manner. The therapist's lack of self-initiated viewing of the logs could be, in part, because of the novelty of the ability to review detailed logs of a session, which is not possible in conventional art therapy. Also, the logs were originally intended to give therapists information for sessions where they are not able to give a client their full attention or when a client chooses to use the ePAD independently (e.g., in a recreational art group or home setting). As therapists were present at all times for the trials, this may have reduced the need to see any external engagement estimates.

Many OAs expressed excitement about the novelty and ease-of-use of ePAD, however, when asked what they liked least about ePAD, many responses related to frustration with some of the functions. In particular, a feature that was intended to make painting seem more realistic was to have the brush "run out" of paint, requiring the client to load more paint on the brush from the paint palate. However, when the paint "ran out" many OAs thought the paint colour was 
1 changing from the one they were using to the colour of the canvas, leading to frustration.

2 Although clients were given the option to use their fingers to paint with ePAD, all participants

3 chose to use a paintbrush instead. This is may be because a tangible paintbrush was a familiar

4 tool that acted as an implicit reminder for OAs about what activity they were doing. Several OAs

5 felt it was challenging to engage with ePAD because the brush did not produce the stoke width

6 they desired. A slider tool was available to change the stoke width, however, OAs did not use it

7 because they either did not know how to or were not aware of it. Hence, having a selection of

8 different (both real and iconic) sized brushes and/or other objects that are familiar to the client

9 could improve the efficiency of ePAD for OAs with dementia.

10 Feedback about effectiveness centered on ePAD's ability to be engaging and its capacity

11 to re-engage clients through prompts. While therapists and OAs expressed that ePAD itself was

12 very engaging, the prompting feature of ePAD was essentially ineffective in enabling

13 engagement. There are many explanations as to why this might be. Therapists did not seem to

14 want to use the prompts and did not set them up unless encouraged by the researcher to do so.

15 The lack of desire to experiment with the prompting feature may have been, in part, due to

16 perceived difficulty, complexity, and reluctance in setting up this feature. These difficulties were

17 exacerbated by wording of the prompts, which did not clearly describe what the prompts did,

18 differences between them, or when they would be used by ePAD. Another reason prompts were

19 not used may have been that encouragement generally evolves as part of the creative process,

20 which is led by the client and then subsequently prompted by the therapist. Thus, a therapist

21 usually cannot predict how he/she will need to prompt because the process is not a procedure that

22 can be gauged ahead of time. Lastly, therapists simply may not have felt that the prompts would

23 be effective in engaging their client. 
ENGAGEMENT IN CREATIVE OCCUPATIONS USING ARTIFICIALLY INTELLIGENT TECHNOLOGY

1

2 intended results. In many cases prompts were not triggered because clients appeared (to ePAD)

3 to be engaged. The majority of prompts that did occur were not effective. For example, one OA

4 became very confused when ePAD gave an audio prompt, which distracted him to the point

5 where the therapist put the process of art-making hold. In another case, the therapist set a prompt

6 that caused the various tools to flash (e.g., the paint brush tool). This prompt was sometimes

7 triggered when the OA participant was considering her next step (e.g., she was not touching or

8 looking at the screen and therefore ePAD thought she was disengaged), which often resulted in

9 distracting, rather than engaging, the client from her task.

11 With the exception of the prompting feature, OAs reported high satisfaction with ePAD. They

12 reported enjoying using the brush to paint and/or add images to the canvas, took pleasure from

13 working with a novel device, and overall, stated that they were pleased with the art they were

14 able to create. Gerdner (2000) explains how art can provide a means for individuals with

15 dementia to reminisce about past events. This was noted with several of the OAs. Rather than

16 creating paintings, one participant preferred to discuss the images that had been added to her

17 toolbox by the therapist; elaborate stories about past life events were discussed during several

18 sessions. This appeared to make the therapist feel unsatisfied with ePAD as it was not engaging

19 the OA in computer-based art, however, the OA appeared to leave the session feeling satisfied.

20 In another case, the way ePAD looked reminded a participant about his past employment in a

21 commercial art firm; this created a lot of reminiscent discussion. Although this type of

22 engagement is not what ePAD was intended to create, it helped to facilitate positive engagement

23 in an interactive process. 
ENGAGEMENT IN CREATIVE OCCUPATIONS USING ARTIFICIALLY INTELLIGENT TECHNOLOGY

Unlike OAs, therapists were unsure about their overall satisfaction with ePAD, and

2 despite high satisfaction ratings from OAs, the therapists remained uncertain as to whether

3 clients were truly satisfied with ePAD. Therapists were satisfied with the overall design and with

4 the way they were able to use ePAD to engage their clients in one-on-one sessions. Many

5 therapists expressed excitement about how valuable it would be to be able to print their final

6 product. Incorporating the therapists' suggestions for improving the prompting and other features

7 of ePAD will be crucial for the next phase of this research.

\section{$8 \quad$ Limitations}

9 There were several limitations inherent to this research. Although the small sample size

10 was sufficient for a preliminary usability study, input from more participants would be valuable.

11 Additionally, only half of the dyads remained in the study until completion; having full data sets

12 would have been beneficial for noting trends in usability and/or engagement over time. While

13 OAs' and therapists' positive comments about the device are encouraging, it must be

14 acknowledged that this could be due (at least in part) to a Hawthorne effect, namely, participants

15 stating what they think the therapist and researchers want to hear because they know they are

16 being observed and want to appear capable, amicable, and polite. This may explain the higher

17 levels of satisfaction reported by the OAs compared to the therapists. Moreover, a Hawthorne

18 effect may have been amplified by the fact that therapists selected clients with whom they had

19 already worked and with whom they had established therapeutic relationships. These possibilities

20 can only be investigated through larger, longer-term trials in more naturalistic art therapy

21 contexts. As this was a prototype device, there were several "bugs" in ePAD's software that

22 negatively impacted the trials. Finally, this study represents feedback from therapists and OAs 


\section{ENGAGEMENT IN CREATIVE OCCUPATIONS USING ARTIFICIALLY INTELLIGENT TECHNOLOGY}

1 from the same facility; it would be interesting to see how data from trials with dyads from other

2 facilities and geographical areas compared.

\section{Conclusion}

$4 \quad$ Measures of usability revealed that all participants found ePAD to be an engaging device,

5 but did not find the prompts effective for enabling engagement. Further research is required to

6 determine what type of prompts could be more effective for engaging OAs with dementia as well

7 as how this device can be used effectively in art therapy contexts. However, regardless of how

8 sophisticated the prompts become, it may be that ePAD's role will be as a tool that fosters

9 accessible and meaningful support to creative art expression for use in dyads in both therapy and

10 non-therapy settings. Technology is becoming more robust, intelligent, and affordable.

11 Combined with cohorts that are increasingly more comfortable using technologies, it is possible

12 that tools such as ePAD will become even more accessible and relevant. In considering the basic

13 philosophy of occupational therapy, ePAD has the potential to enable OAs with dementia engage

14 in a meaningful creative activity, thereby promoting independence and quality of life. Future

15 work includes making the improvements identified in this research, investigating more effective

16 prompting strategies for ePAD, the long-term use of ePAD in more realistic art therapy settings,

17 and eventually, expanding the use of this device from a therapy setting to the home environment

18 for use with family caregivers.

\section{References}

21 American Art Therapy Association. (2011). Art Therapy. from http://www.arttherapy.org/aataaboutus.html

Blunsden, S., Richards, B., Bartindale, T., Jackson, D., Olivier, P., Boger, J., et al. (2009). Design and prototype of a device to engage cognitively disabled older adults in visual 
artwork. Paper presented at the ACM 2nd International Conference on Pervasive Technologies Related to Assistive Environments (PETRA).

Carrillo, M., Dishman, E., \& Plowman, T. (2009). Everyday technologies for Alzheimer's disease care: Research findings, directions, and challenges. Alzheimer's \& Dementia, 5(6), 479-488.

Cummings, J., Miller, B., Christensen, D., \& Cherry, D. (2008). Creativity and dementia: emerging diagnostic and treatment methods for Alzheimer's disease. The International Journal of Neuropschiatric Medicine: CNS Spectrums, 13(2), 1-20.

Folstein, M. F., Folstein, S. E., \& McHugh, P. R. (1975). Mini-Mental State: A practical method for grading the cognitive state of patients for the clinician. Journal of Psychiatric Research, 12(3), 189-198.

Gerdner, L. (2000). Music, art, and recreational therapies in the treatment of behavioural and psychological symptoms of dementia. International Psychogeriatrics, 12(1), 359-366.

Hanson, W., Creswell, J. W., Clark, V., Petska, K., \& Creswell, J. D. (2005). Mixed methods research designs in counselling psychology. Journal of Counselling Psychology, 52(2), 224-235.

Hoey, J., Zutis, K., Leuty, V., \& Mihailidis, A. (2010). A tool to promote prolonged engagement in art therapy: Design and development from art therapist requirement. Paper presented at the ACM SIGCHI Assets Conference.

International Organization for Standardization. (1994). ISO 9241-11: Ergonomic requirements for office work with visual display terminals (VDTs) -- Part 11: Guidance on usability.

Karp, A., Paillard-Borg, S., Wang, H., Silverstein, M., Winblad, B., \& Fratiglioni, L. (2006). Mental, physical and social components in leisure activities equally contribute to decrease dementia risk. Dementia and Geriatrics Cognitive Disorders, 21(2), 65-73.

Kuleba, B. (2008). The integration of computerized art making as a medium in art therapy theory and practice. Drexel University, Philadelphia, PA.

Lubinski, R. (1991). Dementia and communication. Philadelphia: B.C. Decker, Inc.

Mattson, D. (2010). Issues in computerized art therapy assessment. The Arts in Psychotherapy, 37(4), 328-334.

Mihailidis, A., Blunsden, S., Boger, J., Richards, B., Zutis, K., Young, L., et al. (2010). Towards the Development of a Technology for Art Therapy and Dementia: Definition of Needs and Design Constraints. The Arts in Psychotherapy, 37(4), 293-300.

Orr, P. (2005). Technology media: an exploration for "inherent qualities". The Arts in Psychotherapy, 32(1), 1-11.

Peterson, B. (2010). The media adoption stage model of technology for art therapy. Art Therapy: Journal of the American Art Therapy Association, 27(1), 26-31.

Rusted, J., Sheppard, L., \& Waller, D. (2006). A Multi-centre Randomized Control Group Trial on the Use of Art Therapy for Older People with Dementia. Group Analysis, 39(4), 517 536.

Stoukides, J. (2008). Creative and sensory therapies enhance the lives of people with Alzheimer's. Medicine and health, Rhode Island, 91(5), 154-155.

Tullis, T., \& Albert, W. (2008). Measuring the User Experience: Collecting, Analyzing, and Presenting Usability Metrics. Amsterdam, The Netherlands: Elsevier/Morgan Kaufmann.

Waller, D. (2002). The difficulty of being. In D. Waller (Ed.), Arts therapies and progressive illness. London: Routledge. 
2

3

4

5

5

6
7

(

8

9

10

11

12

13

14

15

16

17

18

19

20

21

22

23

24

25

26

27

28

29

30

31

32

33

34

35

36

37

38

39

40

41

42

43

44

45

46

47

48

49

50

51

52

53

54

55

56

57

58

59

60

Wilson, R. J., Rochon, E., Mihailidis, A., \& Leonard, C. (2012). Examining Success of communication strategies used by formal caregivers assisting individuals with Alzheimer's disease during an activity of daily living. Journal of Speech, Language, and Hearing Research, 55(2), 328-341.

6

(1)




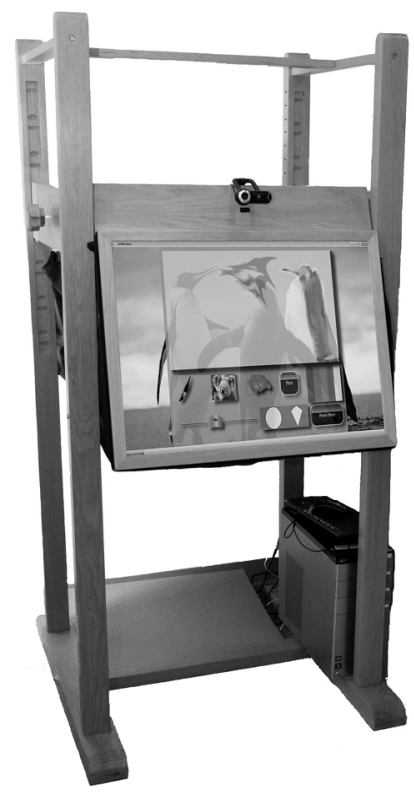

(a)

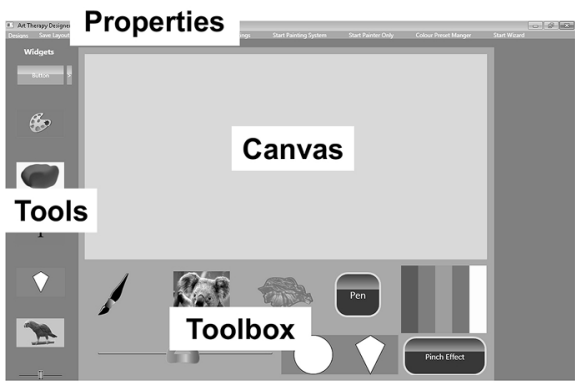

Therapist Interface

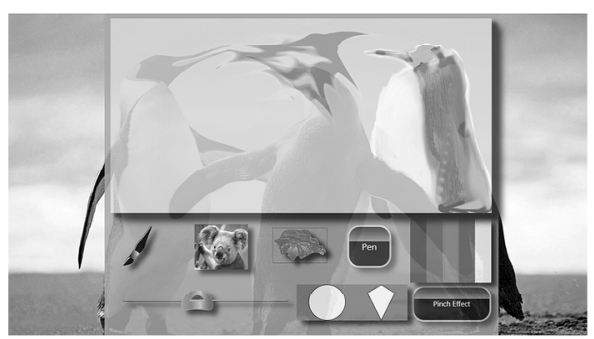

Client Interface

Figure 1. (a) ePAD, which consists of a multi-touch display (screen) and an adjustable wooden easel that contains all of the necessary system electronics. (b) ePAD's interfaces, which consist of the "Therapist Interface" that an art therapist uses to customize the system for their client and the "Client Interface" that an older adult client uses to create his/her artwork.

$203 \times 135 \mathrm{~mm}(300 \times 300 \mathrm{DPI})$ 
Figure 2. Median responses for each question questionnaire administered to therapists after the initial training and setup session, where $1=$ Strongly Disagree, $2=$ Disagree, $3=$ Neutral, $4=$ Agree, $5=$ Strongly Agree (responses $=6$ ) $174 \times 126 \mathrm{~mm}(300 \times 300 \mathrm{DPI})$ 


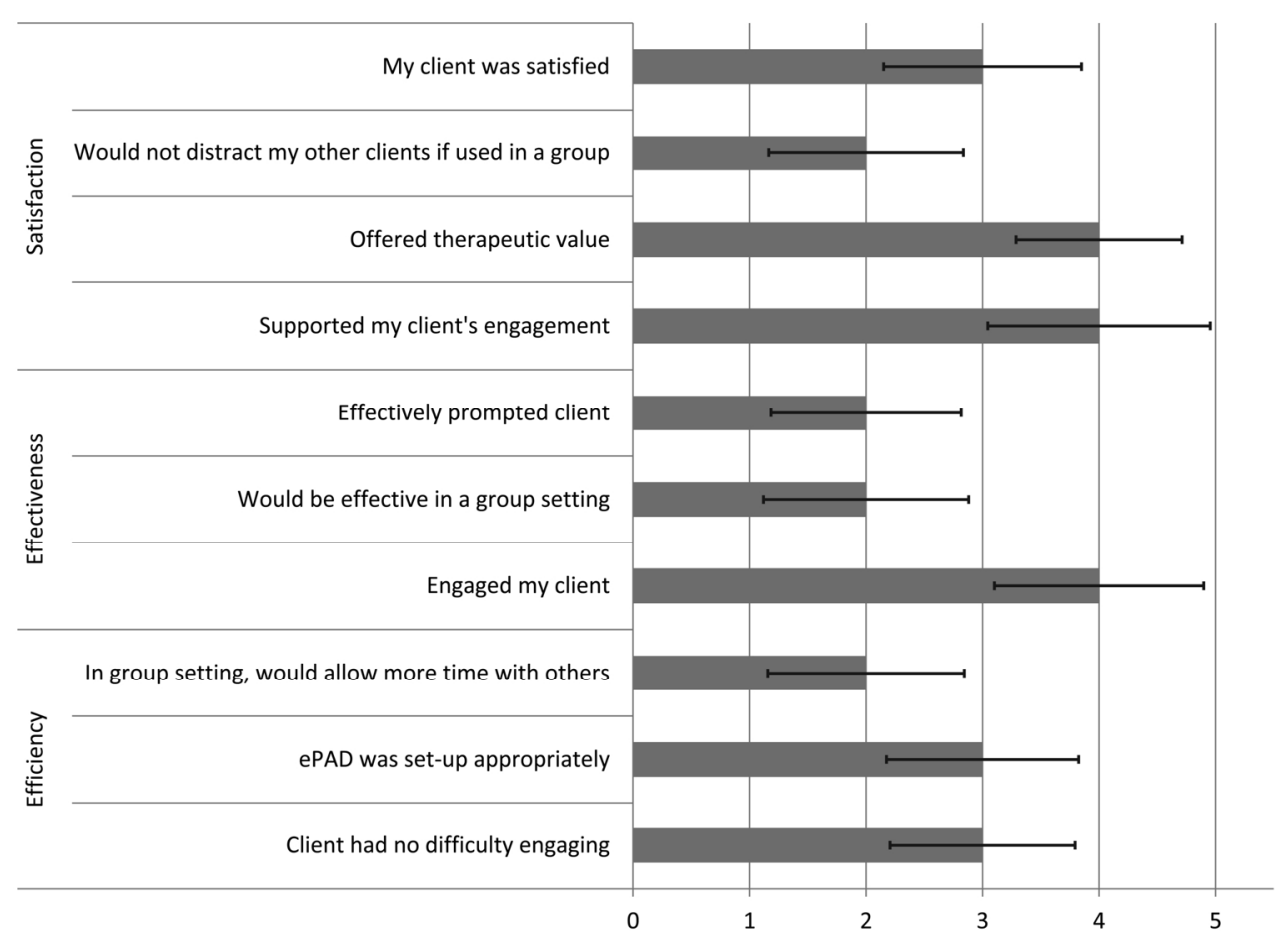

Figure 3. Median responses for each question questionnaire administered to therapists after each session with their client, where $1=$ Strongly Disagree, $2=$ Disagree, $3=$ Neutral, $4=$ Agree, $5=$ Strongly Agree (responses $=21$ ) $174 \times 126 \mathrm{~mm}(300 \times 300$ DPI $)$ 


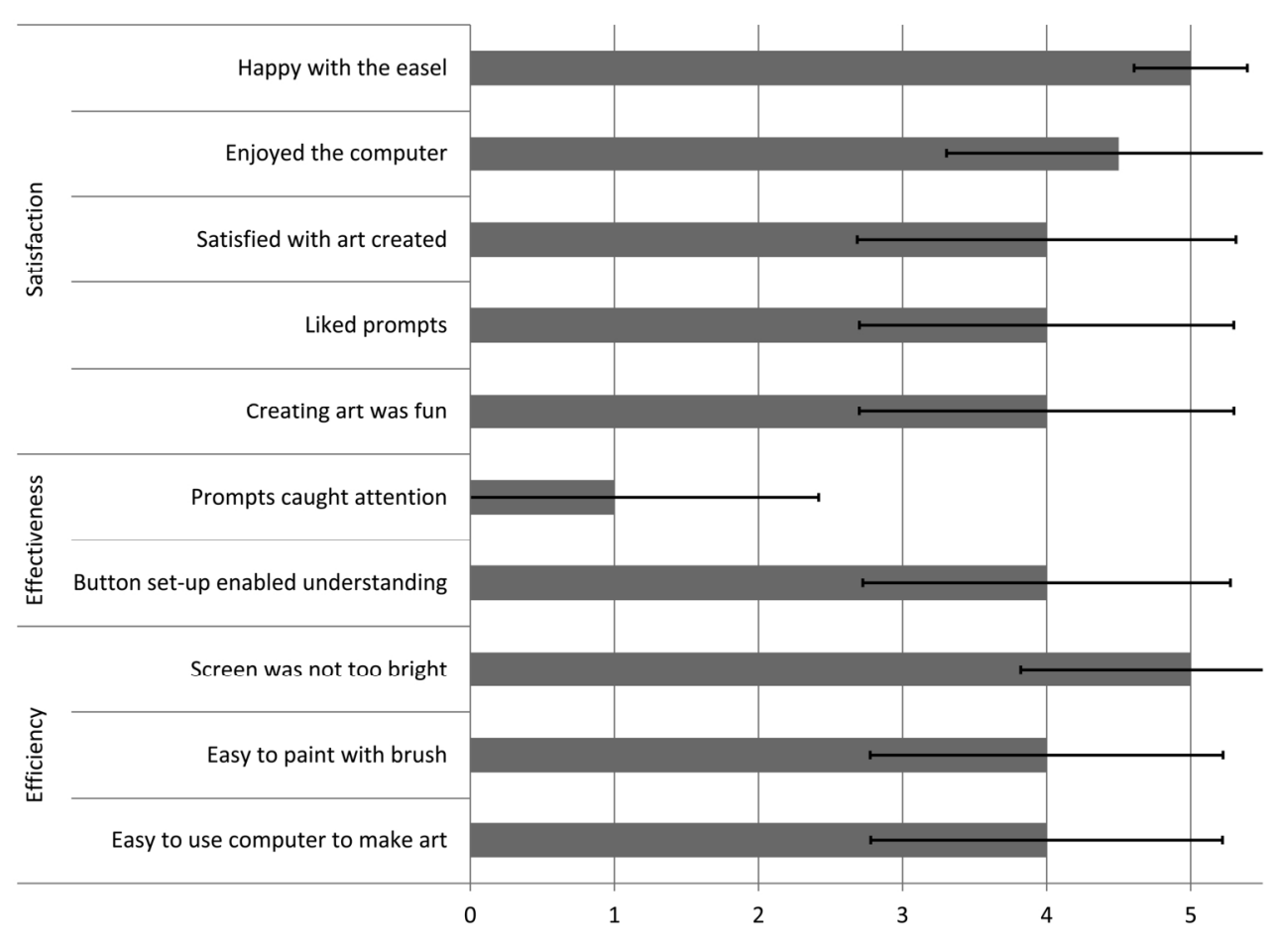

Figure 4. Median responses for each question on questionnaire administered to the older adults with dementia after each testing session, where $1=$ Strongly Disagree, $2=$ Disagree, $3=$ Neutral, $4=$ Agree, $5=$ Strongly Agree (responses $=21)$ $174 \times 126 \mathrm{~mm}(300 \times 300$ DPI) 